\title{
Comparison of different methods of general anaesthesia for controlled hypotension in functional endoscopic sinus surgery (FESS)
}

\author{
Jigisha B Mehta ${ }^{1, *}$, Vandana Parmar ${ }^{2}$, Vrinda Oza $^{3}$ \\ ${ }^{1}$ Senior Resident, ${ }^{2}$ Professor and Head, ${ }^{3}$ Assistant Professor, Dept. of Anaesthesiology, ${ }^{1}$ Government Medical College, Vadodara, \\ Gujarat, ${ }^{2,3}$ Pandit Deendayal Upadhyay Medical College and Hospital, Rajkot, Gujarat, India \\ *Corresponding Author: Jigisha B Mehta \\ Email: jbmehta.28@gmail.com
}

Received: $24^{\text {th }}$ May, 2018

Accepted: $30^{\text {th }}$ August, 2018

\begin{abstract}
Introduction and Aims: Functional Endoscopic Sinus Surgery (FESS) is minimal invasive technique for management of chronic sinusitis. Controlled hypotension is required in FESS for bloodless field, better visualization, reduce operative time and bleeding. Various agents like $\beta$ blockers, $\alpha+\beta$ blockers, magnesium sulfate etc. used to achieve controlled hypotension. The aim of study was to produce controlled hypotension with propofol and isoflurane and to compare intraoperative blood loss, duration of surgery, operative field condition.

Materials and Methods: Sixty patients were assigned in either group by computer generated random number table. Patients in Group A received propofol infusion and in group B received isoflurane in concentration to achieve MBP between 60 to 70 $\mathrm{mmHg}$. Bispectral index value was also maintained between 40 to 60. Statistical difference between two groups was determined using unpaired student $t$ test except for surgical field condition, for which Median test for two populations was used. A " $p$ ” value $<0.05$ was considered as significant.

Result: There was no statistically significant difference in MBP between both groups. Mean intra-operative blood loss was less in group A $(115.17 \pm 12.07 \mathrm{ml})$ compared to group B $(140.5 \pm 15.83 \mathrm{ml})$. Mean duration of surgery was shorter in group A

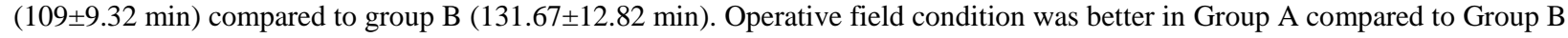
$(p=0.0026)$.

Conclusion: Both propofol and isoflurane effective in producing controlled hypotension, but anaesthesia with propofol is associated with less blood loss, shorter duration of surgery and better surgical field condition compared to isoflurane.
\end{abstract}

Keywords: Bispectral Index, Controlled hypotension, Functional endoscopic sinus surgery, Isoflurane, Propofol.

\section{Introduction}

FESS is a minimal invasive technique for the management of chronic sinusitis and other sinonasal diseases like nasal polyposis. The aim of FESS is to reestablish the drainage and aeration of the paranasal sinuses, while maintaining the natural mucociliary clearance mechanism, and to preserve the normal anatomical structures. ${ }^{1}$ During FESS mucosal bleeding interferes with the visualization of intranasal anatomy, which not only hinders the operation but also increases the incidence of complications. ${ }^{2}$

Controlled hypotension is one of the techniques employed to decrease the amount of bleeding and provide a clear surgical field. ${ }^{3}$ Techniques for controlled hypotension include controlling venous return (elevation of the patient's head), local infiltration of vasoconstrictor (adrenaline) and number of pharmacological interventions including direct acting vasodilator drugs (sodium nitropruside, nitroglycerine, hydralazine); alpha-adrenergic agonists (Clonidine, dexmedetomidine), beta adrenergic antagonists (propranolol, esmolol); alpha and beta adrenergic antagonist (labetalol); magnesium sulfate. Drugs used for anaesthesia can also affect the amount of bleeding and surgical field by vasodilatation and reduction of blood pressure (BP), like high dose of potent inhaled anaesthetics (halothane, isoflurane, sevoflurane); total intravenous anaesthesia using propofol. ${ }^{4}$ Propofol is a derivative of isopropyl phenol that is used in total intravenous anaesthesia. It has antiemetic effects and shorter recovery time. Isoflurane is an inhalation anesthetic with 1.15 Minimum Alveolar Concentration (MAC) that has faster onset after the induction of anaesthesia and also produces hypotension dependent on dose volume. ${ }^{5}$ Primary aim of study is to produce controlled hypotension with propofol and isoflurane. Secondary aims measured are intra operative blood loss, duration of surgery, operative field condition.

\section{Materials and Methods}

The study was carried out after approval of Institutional Ethics Committee. The study was randomized prospective control study. Randomization was done with the use of sealed envelope which contain computer generated random numbers. Odd numbers indicate Group A and even numbers indicate Group B. Table was generated online using site: www.stattrek.com

Double blinding was done to asses blood loss and post operative complications. Anaesthesiologist assessing intra-operative blood loss and surgeon doing FESS were blind to the anaesthestic agent given to the patient. For the purpose of blinding infusion pump containing propofol was attached in all the patients and 
infusion pump and isoflurane vaporizer was covered with opaque cloth so that surgeon and anaesthesiologist assessing blood loss didn't know what patient is receiving for controlled hypotension.

Sixty patients of either sex undergoing FESS, between 12 to 60 year of age and ASA status I, II,III were included in study. Assuming fall in mean blood pressure by $20 \mathrm{mmHg}$ based on previous studies and with power of $90 \%$, alpha error of 0.05 and dropout rate $10 \%$, sample size required was calculated as 30 in both group. Patients with uncontrolled hypertension and diabetes, bleeding disorders, on anticoagulation therapy, coronary artery disease; hepatic, renal, cardiovascular or autonomic dysfunction were excluded from the study. Informed consent was taken from all patients for anaesthesia as well as enrollment in the study. Benefits and likely complications of the techniques used were explained to the patients and their caretaker in understandable language.

After taking patient inside operation room i.v line was secured and Inj. Dextrose Normal Saline (DNS) was started. Standard monitors were applied and baseline parameters like Pulse Rate (PR); Systolic, Diastolic and Mean Blood Pressure (SBP, DBP, MBP respectively), $\mathrm{SpO}_{2}$ were recorded. Bispectral Index (BIS) monitor was also applied and BIS value recorded. Premedication- Inj. Glycopyrrolate $4 \mu \mathrm{g} / \mathrm{kg}$, Inj. Ondansetrone $0.08 \mathrm{mg} / \mathrm{kg}$, Inj. Ranitidine $1 \mathrm{mg} / \mathrm{kg}$, Inj. Fentanyl $1 \mu \mathrm{g} / \mathrm{kg}$ was given. All the patients were preoxygenated with $100 \%$ oxygen for 3 minutes. Induction was done with Inj. Sodium thiopentone $5-7 \mathrm{mg} / \mathrm{kg}$ and inj. Succinylcholine $2 \mathrm{mg} / \mathrm{kg}$ was given to facilitate intubation. Oral intubation was done with proper sized cuffed endotracheal tube and it was fixed after confirming bilaterally equal air entry. Loading dose of Inj. Vecuronium $0.08-0.1 \mathrm{mg} / \mathrm{kg}$ was given to all the patients. Oropharyngeal packing was done in all the patients with saline socked throat pack. At this point patients were enrolled in group according to the number in envelope given to anaesthesiologist. Odd numbers indicate Group A and even numbers indicate Group B.

Group A: Patients of this group received oxygen $\left(\mathrm{O}_{2}\right)$, nitrous oxide $\left(\mathrm{N}_{2} \mathrm{O}\right)$ and propofol infusion for maintenance of anaesthesia. Propofol infusion was started at the rate of $12 \mathrm{mg} / \mathrm{kg} / \mathrm{hr}$ for initial 10 minutes following intubation, then decreased to $10 \mathrm{mg} / \mathrm{kg} / \mathrm{hr}$ for next $10 \mathrm{~min}$, and continued at the rate of $8 \mathrm{mg} / \mathrm{kg} / \mathrm{hr}$ throughout surgery. Infusion rate was adjusted to maintain MBP between 60 to $70 \mathrm{mmHg}$. Propofol infusion was discontinued 10 minutes before completion of surgery.

Group B: Patients of this group received $\mathrm{O}_{2}, \mathrm{~N}_{2} \mathrm{O}$ and isoflurane throughout surgery. Concentration of isoflurane was adjusted to achieve MBP between 60 to $70 \mathrm{mmHg}$.

Throughout intra-operative period BIS value was maintained between 40 to 60 in both the group.
In both the group neuromuscular blockade was maintained with intermittent dose of Inj. Vecuronium $1 / 5^{\text {th }}$ of the bolus dose. Intra venous fluid (DNS and RL) $4 \mathrm{ml} / \mathrm{kg} /$ hour was given. After completion of surgery, throat pack was removed and residual neuromuscular paralysis was reversed with Inj. Neostigmine 0.05 $\mathrm{mg} / \mathrm{kg}$ and inj. Glycopyrrolate $8 \mu \mathrm{g} / \mathrm{kg}$. Extubation was done after attaining spontaneous and adequate respiration.

Intraoperatively all parameters (pulse, SBP, DBP, $\mathrm{MBP}, \mathrm{SpO}_{2}$ ) were recorded before intubation, immediately after intubation, $5 \mathrm{~min}, 10 \mathrm{~min}, 15 \mathrm{~min}, 20$ min, $30 \mathrm{~min}$ and then every 15 minute interval. Blood loss, visibility of operative field and duration of surgery were noted. Evaluation of visibility of the operative field during surgery was done by asking surgeon and grading was noted.

Fromme-Boezaart surgical field grading.

1. Grade 0: no bleeding

2. Grade 1: slight bleeding- no suctioning of blood required.

3. Grade 2: slight bleeding- occasional suctioning required. Surgical field not threatened.

4. Grade 3: slight bleeding-frequent suctioning required. Bleeding threatens surgical field a few seconds after suction is removed.

5. Grade 4: moderate bleeding- frequent suctioning required. Bleeding threatens surgical field directly after suction is removed.

\section{Measurement of Blood Loss}

Measurement of blood loss during surgery was done directly from the collection into the calibrated bottle of suction machine and number of blood socked gauze piece (surgical pattie) used during surgery.

A test was done to measure how much amount of blood is shocked by surgical pattie. Solution containing methylene blue and normal saline was placed into clearly marked graded cylinder. Surgical pattie was then placed into the fluid until it was $100 \%$ saturated. Then change in the volume of cylinder was measured. The mean of 5 separate trials was calculated and was determined to be mean absorptive value for $100 \%$ saturation. The same test was repeated for $50 \%$ and $25 \%$ saturation of surgical pattie and mean absorptive value for $50 \%$ and $25 \%$ was determined.

Mean absorptive value for $100 \%$ saturation of surgical pattie- $2 \mathrm{ml}$

Mean absorptive value for $50 \%$ saturation of surgical pattie- $1 \mathrm{ml}$

Mean absorptive value for $25 \%$ saturation of surgical pattie- $0.5 \mathrm{ml}$

All the data were recorded in Microsoft Excel 2007. Mean as well as Standard Deviation (SD) were calculated. Statistical difference between two groups was determined using unpaired student $t$ test except for surgical field condition. For it Median test for two populations was used. A "p" value $<0.05$ was 
considered significant and "p" value $<0.001$ was considered highly significant.

\section{Result and Discussion}

Both the groups were comparable in demographic data [Table 1]. Graph 1 shows pulse rate in both the groups. No statistically significant difference was noted in pulse rate at any time point. Graph 2 shows mean blood pressure in both the groups. There was no statistically significant difference in mean blood pressure at different time point except from 10 to 30 min. There was statistically significant difference in mean intra operative blood loss between both group $(\mathrm{p}<0.05)$ with less blood loss in group A $(115.17 \pm 12.07$ $\mathrm{ml})$ than group B $(140.5 \pm 15.83 \mathrm{ml})$ [Graph 3]. Graph 4 shows mean duration of surgery. There was statistically significant difference in mean duration of surgery $(\mathrm{p}<0.05)$ with shorter surgery time in group $A$ $(109 \pm 9.32 \mathrm{~min})$ compared to Group B $(131.67 \pm 12.82$ min). Graph 5 shows operative field condition. Statistically significant difference was noted in operative field condition $(\mathrm{p}=0.0026)$ with better surgical field condition in group A compared to group B.

Sinusitis is an important cause of morbidity and may exacerbate chest disease. ${ }^{6}$ Good surgical field visibility is one of the basic prerequisites for a precise and safe otolaryngological operation, and the main obstacle to good visibility is excessive perioperative bleeding. Of the main methods of reducing bleeding during functional endoscopic sinus surgery (FESS) is the use of controlled hypotension. ${ }^{7}$ Controlled hypotension is defined as a reduction of the systolic blood pressure to $80-90 \mathrm{~mm} \mathrm{Hg}$, reduction of mean arterial pressure (MAP) to $50-65 \mathrm{~mm} \mathrm{Hg}$ or $30 \%$ reduction from baseline MAP. ${ }^{8}$ Various studies have been done to compare different drugs for controlled hypotension in FESS. We decided to do comparision of propofol with isoflurane for controlled hypotension in functional endoscopic sinus surgery. In our study MAP was maintained between 60 to $70 \mathrm{mmhg}$. Bispectral index monitoring was also done and BIS value was maintained between 40 to 60 . EEG based BIS monitor has proven to be useful indicator of anaesthetic depth. ${ }^{9}$

In our study both the drugs were equally effective in achieving controlled hypotension but there was less blood loss in patients receiving propofol compared to isoflurane. Isoflurane reduces the arterial pressure by reducing systemic vascular resistance; thus, despite low blood pressure, capillary bleeding increases. ${ }^{10}$ Propofol also reduces the vascular resistance, but in contrast to isoflurane, propofol may either reset or inhibit the baroreflex and reduces the tachycardia response to hypotension from decreased systemic vascular resistance..$^{11}$ In addition, when propofol is continually infused, it causes reduction in the cerebral perfusion pressure, which regulates the cerebral blood flow. ${ }^{12}$ The ethmoid, sphenoid, frontal sinuses are supplied by the branches of internal carotid artery: the ethmoidal and supraorbital artery. ${ }^{13}$ The internal carotid artery is a division of cerebral circulation. Therefore, reduced bleeding can be expected due to the reduced blood flow around the paranasal sinuses.

In addition duration of surgery was also shorter in patients receiving propofol and surgical field condition was better in patients receiving propofol compared to isoflurane. We have not measured time to recovery, amount of isoflurane and propofol used and costeffectiveness.

In study done by Behzad Kazemi Haki, Javad Eftekhari ${ }^{14}$ et al (2014) one group received propofol infusion and other group received isoflurane for maintenance of anaesthesia. Mean heart rate was $69 \pm 14$, SBP was $88.5 \pm 6.92$ and DBP was $63.12 \pm 7.38$ in patients receiving propofol infusion while mean heart rate was $73 \pm 17$, SBP was $91.04 \pm 5.15$ and DBP was $57.51 \pm 6.36$ in patients receiving isoflurane ("p" values: HR-0.74, SBP- 0.086, DBP-0.13). So they concluded that there was no statistical difference in respect of hemodynamic variable between two groups which is similar to our study.

In our study blood loss was less in propofol group compared to isoflurane group. Similar to our study, blood loss was less in propofol group compared to isoflurane group in study done by Shideh Marzban, Soudabeh Haddadi ${ }^{15}$ et al (2013) on comparision of surgical conditions between propofol and isoflurane anaesthesia for Endoscopic Sinus Surgery (ESS). In their study one group $(n=22)$ received propofolremifentanil and second group $(\mathrm{n}=22)$ received isoflurane plus remifentanil infusion. Amount of blood loss was $155 \mathrm{ml}$ in propofol group and $291.3 \mathrm{ml}$ in isoflurane group $(\mathrm{p}=0.003)$. The average surgical duration (minute) was $124.31 \pm 24.11$ minutes in Isoflurane group and $96.36 \pm 28.12$ minutes in propofol group $(\mathrm{P}=0.001)$. They concluded that there was less bleeding with propofol compared to isoflurane and duration of surgery was less during propofol anaesthesia, which is comparable to our study.

Ghodrati MR, Zadimani AR ${ }^{16}$ studied Comparison of blood loss in septorhinoplasty with two different anesthetic technique, propofol and isoflurane. Patients were randomly assigned into two groups (30 patients in each group). After similar induction of anaesthesia in all patients, Group I patients received propofol with remifentanil and Group II patients received isoflurane with remifentanil for maintenance of anaesthesia. The bleeding, surgeons' satisfactions from surgical field and hemodynamic parameters were noted and compared. They concluded that anaesthesia with Propofol result in less blood loss and higher surgeons satisfaction in septorhinoplasty compared with isoflurane anaesthesia.

In contrast to our study, Sarvanan P Ankichetty, Manickam Ponniah ${ }^{17}$ et al (2011) studied comparison between propofol and isoflurane for controlled hypotension in functional endoscopic sinus surgeries 
found no statistically significant difference in mean blood loss and surgical field condition between two groups.

Hassani V, Farhadi $\mathrm{M}^{18}$ et al studied comparison of amount of blood loss in propofol-remifentanil versus isoflurane remifentanil anaesthetic Procedures in Functional Endoscopy Sinus Surgery (FESS). Fourty six patients of ASA status I undergoing endoscopic sinus surgery, were selected and then randomly assigned into two groups of 23 patients each. In both group the induction of anaesthesia was done by propofol, remifentanil and cis-atracarium. Maintenance of anaesthesia in first group was done by using propofol and remifentanil infusion. In the second group maintenance of anaesthesia was done by using isoflurane and remifentanil infusion. They concluded that there was no statistically significant difference in amount of blood loss between two groups.

Table 1: Demographic data

\begin{tabular}{|l|c|c|c|c|}
\hline & Group A & Group B & P-value & Significance \\
\hline Age & $40.23 \pm 13.47$ & $36.1 \pm 14.66$ & 0.26 & Not significance \\
\hline Weight & $59.4 \pm 7.19$ & $57.73 \pm 8.11$ & 0.403193 & Not significance \\
\hline Sex Male: Female & $19: 11$ & $15: 15$ & & \\
\hline
\end{tabular}

Graph 1: Intra-operative pulse rate changes

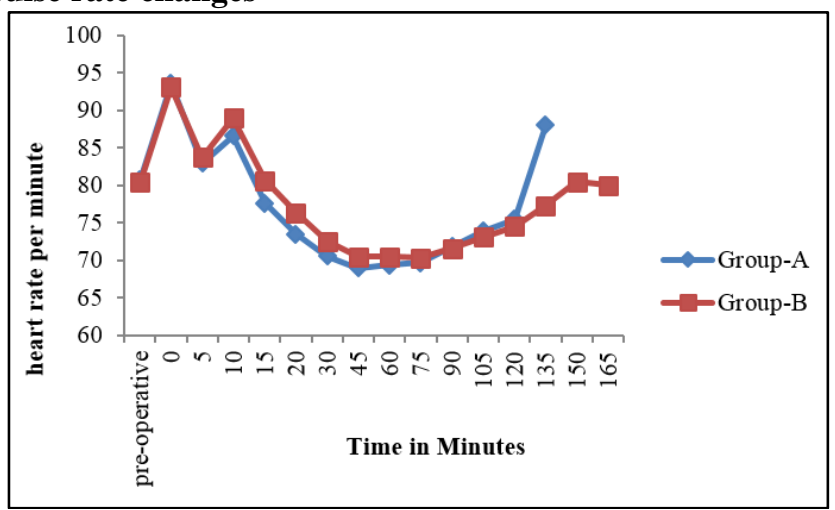

Graph 2: Intra-operative mean blood pressure changes

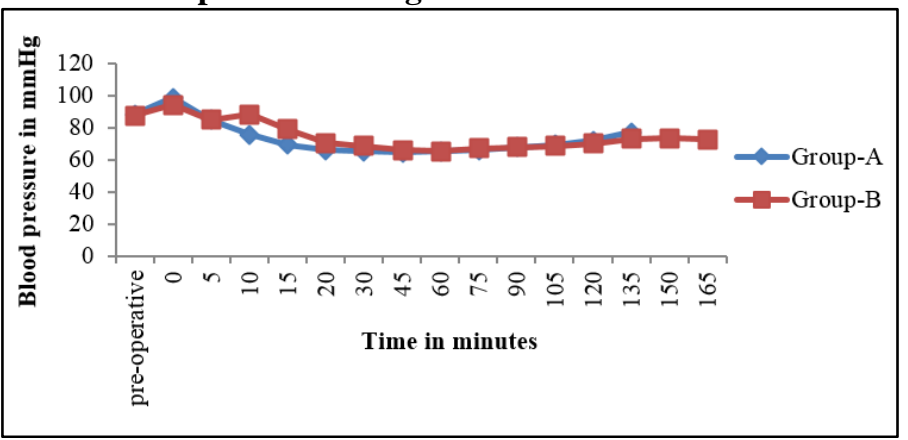

Graph 3: Intra-operative blood loss

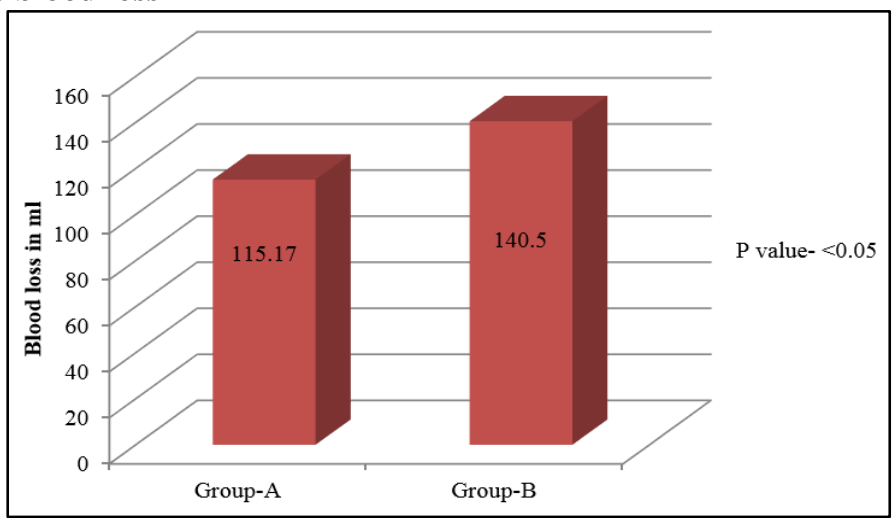




\section{Graph 4: Duration of surgery}

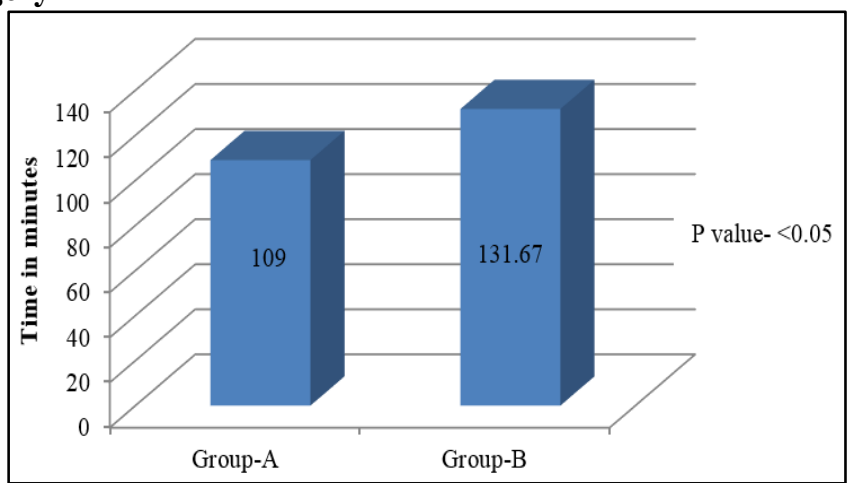

\section{Graph 5: Operative field condition}

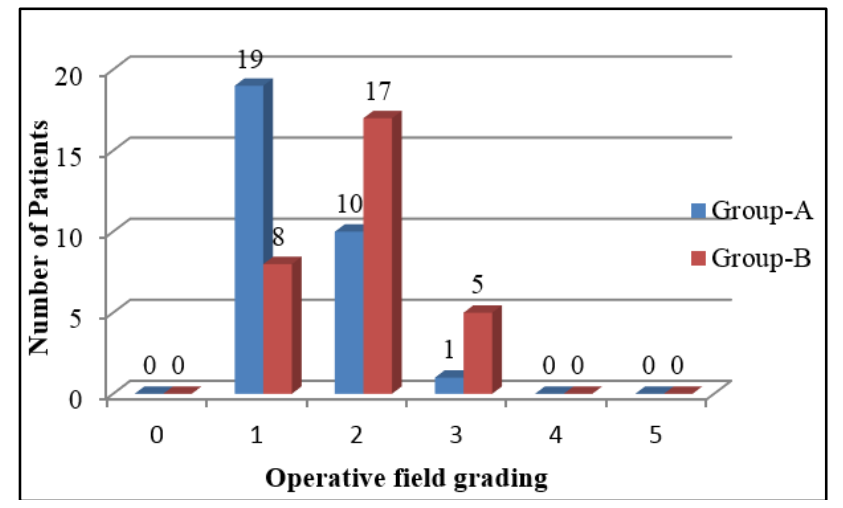

\section{Conclusion}

From our study, we conclude that both propofol and isoflurane effective in producing controlled hypotension in Functional endoscopic sinus surgery, but anaesthesia with propofol is associated with less blood loss, short duration of surgery and better surgical field condition.

\section{Acknowledgement}

We are very grateful to entire ENT department of P.D.U Medical College and hospital, Rajkot for their support.

\section{Conflicts of Interest: None.}

\section{References}

1. Stammberger H, Posawetz W. Functional endoscopic sinus surgery. Concept, indications and results of the Messerklinger technique. Eur Arch Otorhinolaryngol. 1990;247:63-76.

2. Stankiewicz JA. Complications of endoscopic sinus surgery. Otolaryngol Clin North Am. 1989;22:749-758.

3. Cincikas D, Ivaskevicius J. application of controlled arterial hypotension in endoscopic rhinosurgery. Medicina (Kaunas). 2003;39:852-859.

4. Hugo VA, Edward DM. Deliberate hypotension. In: Miller RD, ed. Anaesthesia. Philadelphia: Churchill Livingstone, 2000;1473-1479.

5. Stuart A. Forman, Yumi Ishizawa, Inhaled Anesthetic Pharmacokinetics: Uptake, Distribution, Metabolism, and Toxicity. In: Ronald D. Miller R. Miller, editor. Miller's
Anesthesia, $8^{\text {th }}$ ed. Philadelphia: Elsevier; 2015. p. 63869.

6. Slavin RG. Sinusitis in adults. J Allergy Clin Immunol. 1988;81:1028-1032.

7. Jarosław Miłon'ski, Hanna Zielin'ska-Bliz'niewska, Wojciech Golusin'ski, Joanna Urbaniak, RafałSoban'ski, JurekOlszewski. Effects of three different types of anaesthesia on perioperative bleeding control in functional endoscopic sinus surgery. Eur Arch Otorhinolaryngol. 2013;270:2045-2050.

8. C.S. Degoute, "Controlled hypotension: a guide to drug choice," Drugs. 2007;67:1053-1076.

9. Schraag S, Bathner U, Gajraj R. The performance of electroencephalogram bispectral index and auditory evoked potential index to predict the loss of consciousness during propofol infusion. Anesth Analg. 1999;89:1311.

10. Ebert TJ, Muzi M, Berens R, Goff D, Kampine JP. Sympathetic responses to induction of anaesthesia in humans with propofol or etomidate. Anesthesiol. 1992;76:725-733.

11. Blackwell KE, Ross DA, Kapur P, Calcaterra TC. Propofol for maintenance of general anaesthesia: a technique to limit blood loss during endoscopic sinus surgery. Am J Otolaryngol. 1993;14:262-266.

12. Haberer JP, Audibert G, Saunier CG, Muller C, Laxenaire MC, Hartemann D. Effect of propofol and thiopentone on regional blood flow in brain and peripheral tissues during normoxia and hypoxia in the dog. Clin Physiol. 1993;13:197-207.

13. Činčikas D, Ivaškevičius J, Martinkènas JL, Balseris S. A role of anesthesiologist in reducing surgical bleeding in endoscopic sinus surgery. Medicina (Kaunas). 2010;46:730-734. 
14. Kazemi Haki B, Eftekhari J, Alizadeh V, Tizro P. Comparison of Hemodynamic Stability, Bleeding, and Vomiting in Propofol-Remifentanil and IsofluraneRemifentanil Techniques in Septorhinoplasty Surgery; Propofol-Remifentanil and Isoflurane-Remifentanil. Jentashapir J Health Res. 2014;5:125-130.

15. Marzban S, Haddadi S, Mahmoudi Nia H, Heidarzadeh A, Nemati S, Naderi Nabi B. Comparison of Surgical Conditions During Propofol or Isoflurane Anaesthesia for Endoscopic Sinus Surgery. Anesthesiol Pain Med. 2013;3:234-238.

16. Ghodrati MR, Zadimani AR. Comparison of Blood loss in Septorhinoplasty with two different Anesthetic Technique; Propofol or Isoflurane. Anesthesiol Pain. 2011;2:1-8

17. Ankichetty SP, Ponniah M, Cherian VT, Thomas S, Kumar K. Comparison of total intravenous anaesthesia using propofol and inhalational anaesthesia using isoflurane for controlled hypotension in functional endoscopic sinus surgery. J Anaesth Clin Pharmacol. 2011;27:328-332.
18. Hassani V, Farhadi M, Daneshi A, Naser Nejad S, Koochak M, Khan Niarak E. Comparison of Bleeding Extent in Propofol-Remifentanil Versus Isoflouran Remifentanil Anesthetic Procedures in Functional Endoscopy Sinus Surgery(FESS) in Rasoul-e-Akram Hospital. J Med Sci. 2004;11:737-742.

How to cite this article: Mehta J. B, Parmar V, Oza V. Comparison of different methods of general anaesthesia for controlled hypotension in functional endoscopic sinus surgery (FESS). Indian J Clin Anaesth. 2018;5(4):523-528. 